\title{
Efficient Method for Recycling Silica Materials from Waste Powder of the Photonic Industry
}

\author{
Liang-Yi Lin and Hsunling Bai* \\ Institute of Environmental Engineering, National Chiao Tung University, Hsinchu 300, Taiwan \\ Supporting Information
}

ABSTRACT: An efficient and economic approach is proposed for the fast and direct recovery of silica materials from photonic waste powder. Unlike the conventional alkaline fusion method for the extraction of silica from waste materials, this method possesses advantages of a rapid and low-energyconsumed process with total recovery yield. The obtained mesoporous silica material, denoted as MCM-41(DU)-F, was recovered directly from photonic waste powder at room temperature with the assistance of cationic surfactant, hydrofluoric acid, and ammonia hydroxide. The recycled MCM41(DU)-F with a high specific surface area $\left(788 \mathrm{~m}^{2} / \mathrm{g}\right)$, ordered mesoporous structure $(4.5 \mathrm{~nm})$, and large pore volume $\left(1.1 \mathrm{~cm}^{3} / \mathrm{g}\right)$ was used as support of tetraethylenepentamine (TEPA) for the capture of $\mathrm{CO}_{2}$ from a flue gas stream. The results demonstrated that TEPA-impregnated MCM-41(DU)-F had an adsorption capacity of $120 \mathrm{mg}$ of $\mathrm{CO}_{2} / \mathrm{g}$ of adsorbent. This is higher than the amount adsorbed by TEPA-MCM-41(NaSi) made from pure chemicals $\left(113 \mathrm{mg}\right.$ of $\mathrm{CO}_{2} / \mathrm{g}$ of adsorbent $)$ and TEPA-MCM-41(AF) made from alkaline fusion (112 $\mathrm{mg}$ of $\mathrm{CO}_{2} / \mathrm{g}$ of adsorbent) under the same testing conditions. This novel recycling process, which can improve cost effectiveness for the mass

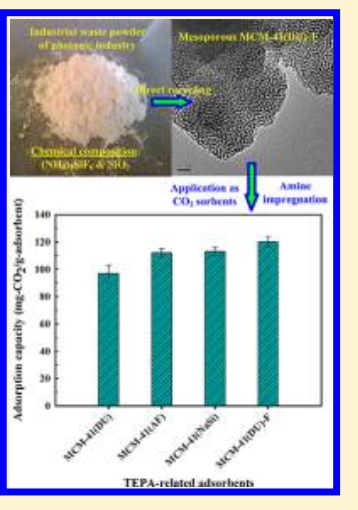
production of valuable mesoporous silica materials from cheap and abundant resources through convenient preparation steps, is surely beneficial from the viewpoint of economical use of photonic industrial waste powder.

\section{INTRODUCTION}

A significant quantity of silicon $(\mathrm{Si})$-containing waste powder is generated from photonic and semiconductor industries because of the growing markets. ${ }^{1}$ The waste powders have problems of treatment and disposal because of their light-density and bulky volume. In general, the primary components contained in photonic waste powder are sub-micrometer $\mathrm{SiO}_{2}$ particles as well as F- and N-related compounds. ${ }^{1}$ The small particle sizes of such waste powders induce harmful effects on humans if not properly treated.

Resource recovery is one of the effective strategies in waste management, which can reduce the need for additional landfill space and conserve natural resources. The recovery and applications of silicon-containing solid wastes, such as coal fly ash and rice husk ash, have been successfully conducted, which include adsorbents for heavy metals in wastewater, ${ }^{2}$ catalyst support for preparing carbon nanotubes ${ }^{3}$ and heterogeneous catalysts, ${ }^{4}$ etc. Recently, coal fly ash has also been shown to be potential sources for the manufacture of porous materials through zeolitization because coal fly ash is primarily composed of $\mathrm{SiO}_{2}$ and $\mathrm{Al}_{2} \mathrm{O}_{3}{ }^{5-9}$ A low-cost zeolite material can be extensively applied for environmental protection use.

Ordered mesoporous silica has attracted a great deal of attention since the first discovery of M41S by Mobil Oil Corporation. ${ }^{10}$ Mesoporous silica with a high surface area, tunable pore structure, and unique surface chemistry via functionalization provides potential applications in adsorption and catalytic processes, etc. ${ }^{11,12}$ In particular, there has been a growing interest in the utilization of ordered mesoporous silica on environmental protection. For example, the control of abundant carbon dioxide by amine-functionalized mesoporous silica is one of the most challenging issues in recent years. ${ }^{13}$ Generally, mesoporous silica was mainly made using pure chemical sources, such as silicon alkoxides, ${ }^{14}$ colloidal silica, ${ }^{15}$ and sodium silicate. ${ }^{16}$ However, large demands of silicon sources would lead to the fast drain and shortage of natural resources.

There have been studies on the conversion of either coal fly ash or rice husk ash into mesoporous silica, where alkaline fusion and/or hydrothermal treatments are employed for the effective extraction of silica source from the solid wastes. ${ }^{17-20}$ Chang and co-workers ${ }^{21}$ first reported the synthesis of mesoporous aluminosilicate, which was hydrothermally processed at $115^{\circ} \mathrm{C}$ for $150 \mathrm{~h}$ using the supernatant extracted from coal fly ash via the alkaline fusion method. Later on, Hui and Chao ${ }^{22}$ proposed a simplified procedure, where coal fly ash was treated with sodium hydroxide solution under mild conditions of $100{ }^{\circ} \mathrm{C}$ for $4.5 \mathrm{~h}$ to obtain the supernatant.

Our prior study ${ }^{1}$ demonstrated the possibility of extracting silicate supernatant from photonic waste powder, which was obtained from the exhaust of the chemical vapor deposition (CVD) process in thin-film transistor-liquid crystal display (TFT-LCD) plants. The alkaline fusion method was employed in our prior study, and the obtained supernatant was further used for manufacturing silica materials. However, the

Received: October 21, 2011

Revised: January 21, 2013

Accepted: March 22, 2013

Published: March 22, 2013 
disadvantages of the alkaline fusion method for extracting the silicon from solid wastes are the long processing period $(\sim 24$ h) and the low silicon recovery yield $(20-33 \%) .{ }^{20,22}$ Thus, the industrial application of this fusion process would have certain limitations. An efficient and low-energy method for the total recycling of photonic waste powder into valuable mesoporous silica must be developed.

In this study, a facile and low-energy-consumed approach on the direct use of industrial photonic silicate waste for complete recycling into silica resources is proposed for the first time. Detailed characterization and formation mechanisms of silica materials via the direct usage of silicate waste powder were investigated and discussed. In addition, an attempt has also been made to evaluate the performance of such inexpensive siliceous materials as supports of adsorbents for $\mathrm{CO}_{2}$ capture. The recycled waste-derived mesoporous silica shows superior $\mathrm{CO}_{2}$ adsorption performance over mesoporous silica MCM$41(\mathrm{NaSi})$ obtained from pure chemical. Therefore, the present study not only proposes a possible route for minimizing the release of hazardous substances into the environment, contributing to the prevention of environmental contamination, but also benefits massive production in highly valuable silicabased sorbents for environmental protection applications, avoiding the fast drain and shortage of natural resources.

\section{EXPRERIMENTAL SECTION}

Recovery of Mesoporous Silica from Photonic Waste Powder. The photonic waste powder was obtained from a TFT-LCD plant, and its chemical composition can be seen in Table S1 of the Supporting Information. Mesoporous silica was synthesized via the direct utilization of raw waste powder as the silica source, and cetyltrimethylammonium bromide (CTAB) was employed as the structure-directing template. The molar composition of the gel mixture was $1 \mathrm{SiO}_{2} / 0.2 \mathrm{CTAB} / x \mathrm{HF} / 12$ $\mathrm{NH}_{4} \mathrm{OH} / 120 \mathrm{H}_{2} \mathrm{O}$ ( $x$ varies with the molar ratio of $\mathrm{HF} / \mathrm{Si}$ ). In a typical procedure, $9.35 \mathrm{~g}$ of waste powder was first dissolved in $137 \mathrm{~mL}$ of deionized (DI) water. Subsequently, a given amount of hydrofluoric acid was added to the above solution. Meanwhile, $7.28 \mathrm{~g}$ of $\mathrm{CTAB}$ was dissolved in $25 \mathrm{~mL}$ of DI water, and then it was added dropwise to the above solution. Then, $32.06 \mathrm{~g}$ of ammonium hydroxide solution was slowly added to promote the hydrolytic condensation of the silicasurfactant mixture. All of the above procedures were performed under continuous stirring. The resulting gel mixture was aged at room temperature for $8 \mathrm{~h}$; the resultant solid was recovered by filtration, washed with DI water, and dried in an oven at $110^{\circ} \mathrm{C}$ for $6 \mathrm{~h}$. Finally, the organic template was removed using a muffle furnace in air at $550{ }^{\circ} \mathrm{C}$ for $6 \mathrm{~h}$. The mesoporous MCM41 synthesized by direct usage of waste powder as the silica source in the absence of hydrofluoric acid was denoted as MCM-41(DU), while the MCM-41 sample synthesized by direct usage of waste powder in the presence of hydrofluoric acid was denoted as MCM-41(DU)-F.

For comparison purposes, the waste-derived mesoporous MCM-41 material as extracted via the alkaline fusion process was also performed. The waste powder was treated with sodium hydroxide at a weight ratio of 1:1.2, and the mixture was then heated at $550{ }^{\circ} \mathrm{C}$ for $1 \mathrm{~h}$ to obtain the fused mass. The activation temperature of $550{ }^{\circ} \mathrm{C}$ has been proven effective in extracting silicon from silicon-containing solid wastes. ${ }^{21}$ The received fused product was then mixed with DI water at a weight ratio of 1:5 with continuous stirring for $24 \mathrm{~h}$. The resulting mixture was then centrifuged to separate the sediment and the supernatant. The obtained silicate supernatant was used as the silica source for the synthesis of MCM-41, with detailed procedures described in our prior study. ${ }^{1}$ The molar composition of the gel mixture was $1 \mathrm{SiO}_{2} / 0.2 \mathrm{CTAB} / 120$ $\mathrm{H}_{2} \mathrm{O} / 0.89 \mathrm{H}_{2} \mathrm{SO}_{4}$. The MCM-41 material using silicate supernatant derived from the alkaline fusion process was denoted as MCM-41(AF). Moreover, the synthesis of MCM-41 using pure chemicals of sodium metasilicate nonahydrate $\left(\mathrm{Na}_{2} \mathrm{SiO}_{3} \cdot 9 \mathrm{H}_{2} \mathrm{O}\right)$ as the silica source was studied as well, which was similar to that described in MCM-41(AF). The obtained material from pure chemicals of $\mathrm{Na}_{2} \mathrm{SiO}_{3} \cdot 9 \mathrm{H}_{2} \mathrm{O}$ was denoted as MCM-41(NaSi).

Application of Recycled Mesoporous Silicas for $\mathrm{CO}_{2}$ Capture. Mesoporous silica adsorbents were functionalized with the amine agent of tetraethylenepentamine (TEPA) at a weight ratio of $1: 1$ in ethanol by the wet impregnation method. The TEPA has been proven as an efficient reagent for the capture of $\mathrm{CO}_{2} \cdot{ }^{13}$ Details of the impregnation procedures and the $\mathrm{CO}_{2}$ adsorption tests are described in the Supporting Information.

\section{RESULTS AND DISCUSSION}

Recycling of Photonic Waste Powder into Mesoporous Silica. Panels a and $\mathrm{b}$ of Figure 1 depict the alkaline

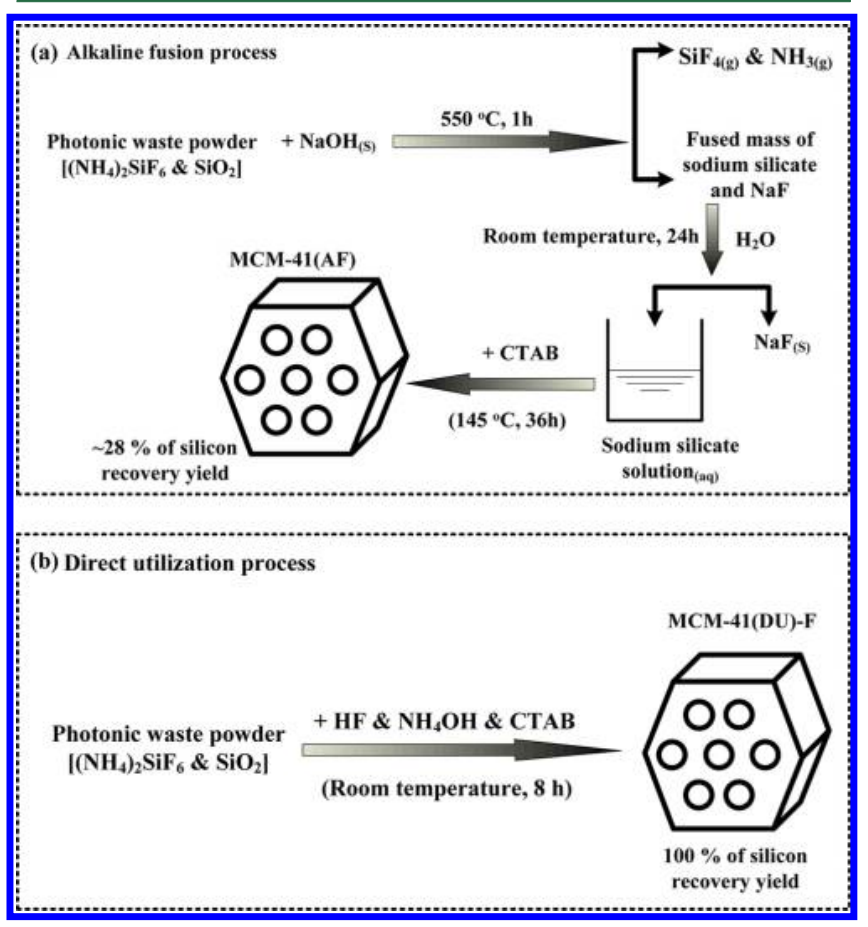

Figure 1. Schematic procedures for the (a) conventional alkaline fusion process and (b) direct utilization process for the recovery of mesoporous silica from the photonic waste powder.

fusion process and the direct utilization process for the recovery of silica materials from the photonic waste powder, respectively. For the alkaline fusion process, the formation mechanism of silica materials from photonic waste powder can be explained by considering the chemical reactions presented in eqs $1-3$. In brief, photonic waste powder consisting of $\left(\mathrm{NH}_{4}\right)_{2} \mathrm{SiF}_{6}$ and $\mathrm{SiO}_{2}$ was decomposed into gaseous silicon tetrafluoride $\left(\mathrm{SiF}_{4}\right)$ and ammonium fluoride $\left(\mathrm{NH}_{4} \mathrm{~F}\right)$ solid residue when the temperature was above $220{ }^{\circ} \mathrm{C}$. ${ }^{1}$ 


$$
\left(\mathrm{NH}_{4}\right)_{2} \mathrm{SiF}_{6(\mathrm{~s})} \stackrel{550^{\circ} \mathrm{C}}{\longrightarrow} \mathrm{SiF}_{4(\mathrm{~g})} \uparrow+2 \mathrm{NH}_{4} \mathrm{~F}_{(\mathrm{s})}
$$

Subsequently, remaining $\mathrm{NH}_{4} \mathrm{~F}$, which is thermally stable up to $850^{\circ} \mathrm{C}$, would react with sodium hydroxide to produce gaseous ammonia and sodium fluoride solid residue.

$$
\mathrm{NaOH}_{(\mathrm{s})}+\mathrm{NH}_{4} \mathrm{~F}_{(\mathrm{s})} \stackrel{550^{\circ} \mathrm{C}}{\longrightarrow} \mathrm{NaF}_{(\mathrm{s})}+\mathrm{NH}_{3(\mathrm{~g})} \uparrow+\mathrm{H}_{2} \mathrm{O}_{(\mathrm{g})} \uparrow
$$

Meanwhile, $\mathrm{SiO}_{2}$ presented in photonic waste powder would also react with sodium hydroxide to produce sodium silicate.

$$
2 \mathrm{NaOH}_{(\mathrm{s})}+\mathrm{SiO}_{2(\mathrm{~s})} \stackrel{550{ }^{\circ} \mathrm{C}}{\longrightarrow} \mathrm{Na}_{2} \mathrm{SiO}_{3(\mathrm{~s})}+\mathrm{H}_{2} \mathrm{O}_{(\mathrm{g})} \uparrow
$$

The obtained sodium silicate was then used as the silica source to synthesize mesoporous MCM-41 in the presence of cationic surfactant of $\mathrm{CTAB}$ via hydrothermal treatment at $145{ }^{\circ} \mathrm{C}$ for 36 h, as shown in Figure 1a. Therefore, the gaseous pollutants of $\mathrm{SiF}_{4}$ and $\mathrm{NH}_{3}$ tend to be formed during the alkaline fusion process for waste recovery.

The photonic waste powder is mainly composed of $85 \%$ $\left(\mathrm{NH}_{4}\right)_{2} \mathrm{SiF}_{6}$ and $15 \% \mathrm{SiO}_{2}$, and the total $\mathrm{Si}$ mass fraction [from $\left(\mathrm{NH}_{4}\right)_{2} \mathrm{SiF}_{6}$ and $\left.\mathrm{SiO}_{2}\right]$ detected by inductively coupled plasma-mass spectrometry (ICP-MS) analysis is $22.4 \%$, as obtained from our prior study. ${ }^{1}$ Silicon recovery yield was determined by the weight ratios of silicon contents in the raw waste powder and the silicon contents in the obtained mesoporous silica materials. It is noted from Figure 1a that there is only $28 \%$ of silicon recovery yield for MCM-41(AF) obtained via the alkaline fusion process. This is similar to the report by Chang and colleagues, who employed the alkaline fusion method for extracting silicon from coal fly ash, and the silicon recovery yield was ca. $32 \%{ }^{21}$ They stated that a sufficient amount of sodium hydroxide and activation time is required for effectively converting silica in the form of quartz and mullite phases into more soluble forms of sodium silicate during the fusion process.

In the present study, the decomposition of $\left(\mathrm{NH}_{4}\right)_{2} \mathrm{SiF}_{6}$ at $550{ }^{\circ} \mathrm{C}$ results in losing silicon species from the release of gaseous silicon tetrafluoride. It is reasonable that the silicon recovery yield would be significantly affected by the loss of silicon species from gaseous silicon tetrafluoride because the photonic waste powder is mainly composed of $85 \%\left(\mathrm{NH}_{4}\right)_{2} \mathrm{SiF}_{6}$ and $15 \% \mathrm{SiO}_{2}$. Therefore, even though MCM-41(AF) with a very high specific surface area can be manufactured, the alkaline fusion process might not be considered as an economic and energy-effective approach because of its low silicon recovery yield, long processing time, gaseous pollution formation, and high operational temperature.

Figure $1 \mathrm{~b}$ reveals the direct utilization process proposed in this study. In a typical process, the photonic waste powder was first treated with hydrofluoric acid. It is expected that $\mathrm{SiO}_{2}$ presented in photonic waste powder would react with hydrofluoric acid to produce hexafluorosilicic acid $\left(\mathrm{H}_{2} \mathrm{SiF}_{6}\right)$, and the chemical reaction is presented as follows:



Subsequently, the hydrolysis of $\left(\mathrm{NH}_{4}\right)_{2} \mathrm{SiF}_{6}$ and $\mathrm{SiF}_{6}{ }^{2-}$ can be accelerated by the addition of ammonium hydroxide.

$$
\begin{gathered}
\left(\mathrm{NH}_{4}\right)_{2} \mathrm{SiF}_{6(\mathrm{aq})}+4 \mathrm{NH}_{4} \mathrm{OH}_{(\mathrm{aq})} \\
\rightarrow \mathrm{Si}(\mathrm{OH})_{4(\mathrm{aq})}+6 \mathrm{NH}_{4} \mathrm{~F}_{(\mathrm{aq})}
\end{gathered}
$$

$$
\begin{aligned}
& 2 \mathrm{H}_{(\mathrm{aq})}^{+}+\mathrm{SiF}_{6}{ }^{2-}(\mathrm{aq}) \\
& \quad \rightarrow 6 \mathrm{NH}_{4} \mathrm{OH}_{(\mathrm{aq})} \\
& \rightarrow \mathrm{Si}(\mathrm{OH})_{4(\mathrm{aq})}+6 \mathrm{NH}_{4} \mathrm{~F}_{(\mathrm{aq})}+2 \mathrm{H}_{2} \mathrm{O}_{(\mathrm{l})}
\end{aligned}
$$

Therefore, the overall reactions for the recovery of silica materials from photonic waste powder in the presence of hydrofluoric acid and ammonium hydroxide can be summarized as follows:

$$
\begin{gathered}
\left(\mathrm{NH}_{4}\right)_{2} \mathrm{SiF}_{6}+\mathrm{SiO}_{2}+6 \mathrm{HF}+10 \mathrm{NH}_{4} \mathrm{OH} \\
\rightarrow 2 \mathrm{Si}(\mathrm{OH})_{4}+12 \mathrm{NH}_{4} \mathrm{~F}+4 \mathrm{H}_{2} \mathrm{O}
\end{gathered}
$$

Also, it is well-known that the ionization of $\mathrm{Si}(\mathrm{OH})_{4}$ produces anion silicate, such as $\mathrm{SiO}(\mathrm{OH})_{3}{ }^{-}$, and the condensation rate can be greatly enhanced using basic catalyst. ${ }^{23}$



The ionization of $\mathrm{Si}(\mathrm{OH})_{4}$ makes silanol more electrophilic and, thus, more susceptible to react with the cationic surfactants of $\mathrm{CTAB}$ by electrostatic force. Finally, the cooperative assembly between the anionic silicate hydrolyzed from $\mathrm{SiF}_{6}{ }^{2-}$ and free charged micelles undergo extensive condensation and polymerization; thus, the mesoporous silica material can be rapidly regenerated at ambient temperature. The novel MCM41(DU)-F material can be obtained from direct use and complete recycling of photonic waste powder as the silica source. The process is simple, fast, and low-energy-consumed, which is more cost-effective when compared to the alkaline fusion process shown in Figure 1a.

Low-angle powder X-ray diffraction (XRD) patterns of MCM-41(DU), MCM-41(DU)-F, and MCM-41(AF) are shown in Figure 2a. The results reveal the presence of the

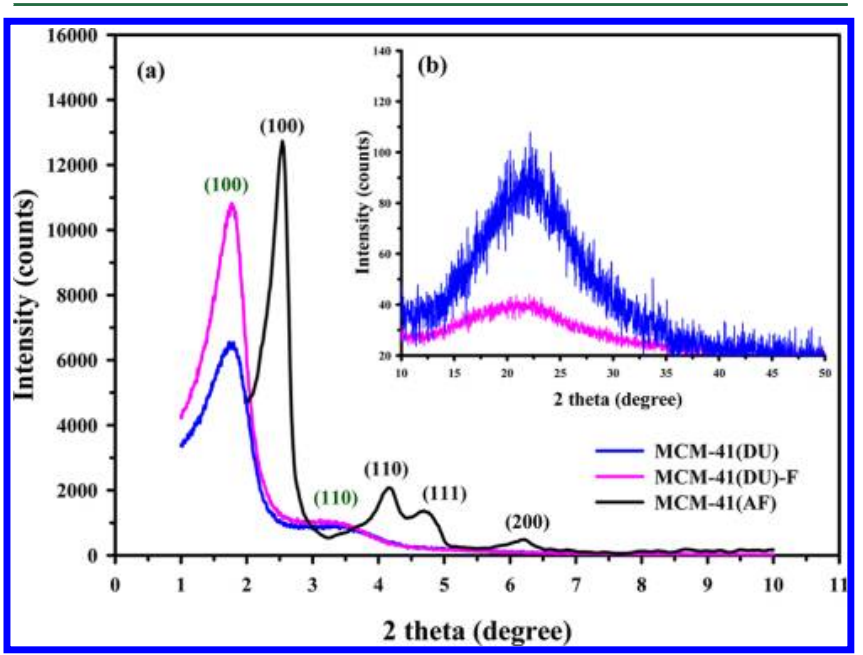

Figure 2. (a) Low-angle XRD patterns of MCM-41(DU), MCM41(DU)-F, and MCM-41(AF) and (b) wide-angle XRD patterns of MCM-41(DU) and MCM-41(DU)-F samples.

hexagonal lattice of the MCM-41(AF) material prepared via the alkaline fusion process, where two well-defined diffraction peaks of (100) and (110) located at $2 \theta$ of $2.5^{\circ}$ and $4.1^{\circ}$ were observed. $^{23}$ Similarly, MCM-41(DU) and MCM-41(DU)-F materials also exhibit two diffraction peaks of (100) and (110) located at $2 \theta$ of $1.7^{\circ}$ and $3.5^{\circ}$, indicating that the silica materials with hexagonal mesostructure can be obtained by direct usage of photonic waste powder as the silica source. It can be observed that MCM-41(DU)-F shows a higher intensity of 


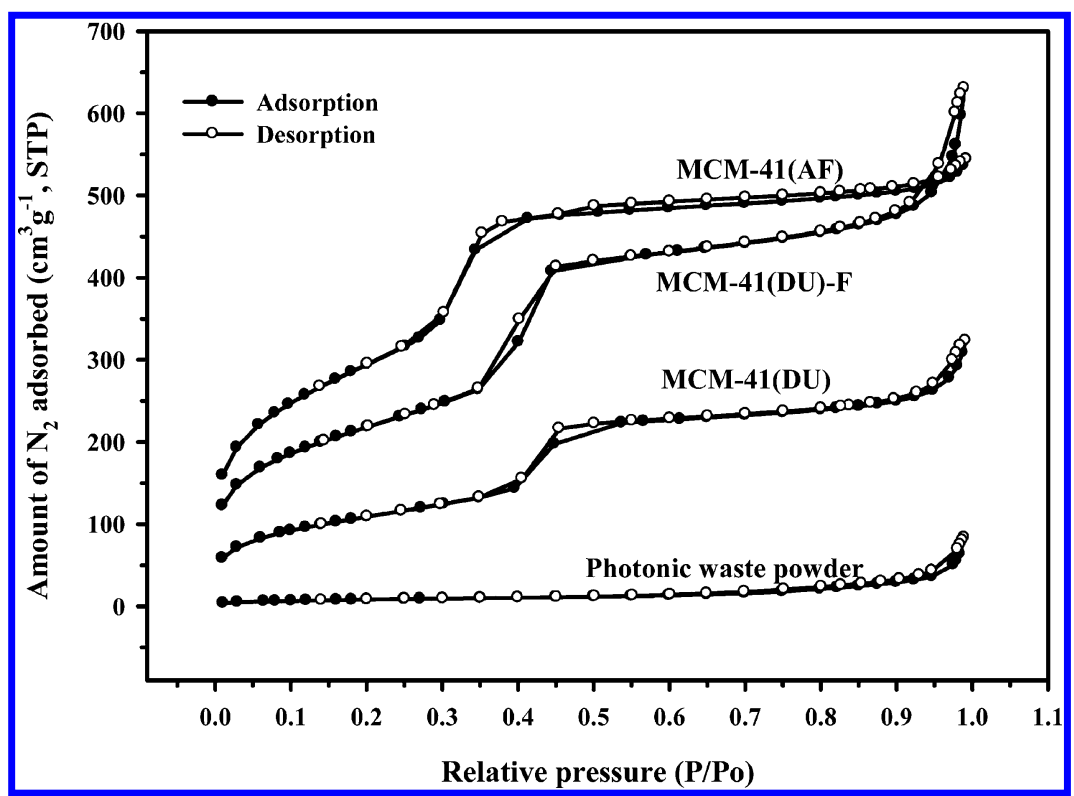

Figure 3. $\mathrm{N}_{2}$ adsorption-desorption isotherms of raw photonic waste powder, MCM-41(DU), MCM-41(DU)-F, and MCM-41(AF) samples.

Table 1. Structural Properties of MCM-41(NaSi) Silica, Waste Powder, and Waste-Derived Mesoporous Siliceous Materials

\begin{tabular}{|c|c|c|c|c|c|c|c|}
\hline sample name & $\begin{array}{l}S_{\mathrm{BET}}{ }^{a} \\
\left(\mathrm{~m}^{2} / \mathrm{g}\right)\end{array}$ & $d_{\mathrm{BJH}}^{b}(\mathrm{~nm})$ & $V_{\mathrm{p}}^{c}\left(\mathrm{~cm}^{3} / \mathrm{g}\right)$ & $\begin{array}{l}R^{d} \\
(\%)\end{array}$ & $\begin{array}{l}\mathrm{N} \text { loading } \\
(\mathrm{mmol} / \mathrm{g})\end{array}$ & $\begin{array}{l}\text { N surface density } \\
\left(\mathrm{N} \text { atom } / \mathrm{nm}^{2}\right)\end{array}$ & $\begin{array}{c}\mathrm{CO}_{2} \text { capacity } \\
(\mathrm{mg} / \mathrm{g})\end{array}$ \\
\hline waste powder & 30 & & 0.07 & & & & \\
\hline MCM-41(AF) & $1060 \pm 13$ & $2.95 \pm 0.05$ & $0.99 \pm 0.02$ & 28 & & & 7 \\
\hline MCM-41(DU) & $391 \pm 7$ & $4.4 \pm 0.04$ & $0.53 \pm 0.01$ & 100 & & & 5 \\
\hline MCM-41(DU)-F & $790 \pm 15$ & $4.5 \pm 0.1$ & $1.10 \pm 0.02$ & 100 & & & 6 \\
\hline MCM-41(NaSi) & 1102 & 3.1 & 1.00 & & & & 7 \\
\hline TEPA-MCM-41(AF) & 55 & & 0.14 & & 10.57 & 6.0 & $112 \pm 3$ \\
\hline TEPA-MCM-41(DU) & 9 & 3.1 & 0.02 & & 10.86 & 16.5 & $97 \pm 6$ \\
\hline TEPA-MCM-41(DU)-F & 36 & 4.0 & 0.16 & & 11.54 & 8.8 & $120 \pm 4$ \\
\hline TEPA-MCM-41(NaSi) & 53 & & 0.15 & & 11.28 & 6.2 & $113 \pm 3$ \\
\hline
\end{tabular}

${ }^{a}$ BET surface area. ${ }^{b}$ Pore diameter calculated by BJH theory. ${ }^{c}$ Pore volume. ${ }^{d} R=$ silicon recovery yield $=$ weight ratios of silicon contents in raw waste powder to silicon contents in mesoporous silica.

(100) reflection than that of MCM-41(DU), which suggests better pore arrangement of MCM-41(DU)-F. On the other hand, there is a broad peak centering at $22^{\circ}$ observed in Figure $2 b$ for MCM-41(DU) and MCM-41(DU)-F, which could be ascribed to the presence of amorphous $\mathrm{SiO}_{2}$ as extra-framework particles. Moreover, it is noticeable that the (100) and (110) reflection peaks of MCM-41(DU) and MCM-41(DU)-F materials shifted to lower degrees compared to those of MCM-41(AF). This implies that MCM-41(DU) and MCM41(DU)-F materials exhibit larger d-spacing values than that of MCM-41(AF).

The $\mathrm{N}_{2}$ adsorption-desorption isotherms of the waste powder, MCM-41(AF), MCM-41(DU), and MCM-41(DU)$\mathrm{F}$ samples are plotted in Figure 3. Apparently, the photonic waste powder revealed a typical type II isotherm of nonporous materials according to the International Union of Pure and Applied Chemistry (IUPAC) classification. On the other hand, MCM-41(AF), MCM-41(DU), and MCM-41(DU)-F samples clearly show capillary condensation steps with $\mathrm{H} 1$ hysteresis loops at a relative pressure of $p / p_{0}=0.25-0.45$, which is attributed to the textural mesoporosity and corresponded to capillary condensation of $\mathrm{N}_{2}$ molecules within the interparticle pores. ${ }^{24}$ The less steep condensation for the MCM-41(DU) sample indicates a less degree of uniform mesostructure compared to that of MCM-41(AF) and MCM-41(DU)-F samples.

Furthermore, the capillary condensation steps of MCM41(DU) and MCM-41(DU)-F samples tend to shift toward higher values of relative pressure, indicating that MCM41(DU) and MCM-41(DU)-F samples possess a larger pore diameter. This result is further confirmed by the BarrettJoyner-Halenda $(\mathrm{BJH})$ pore size distribution shown in Figure S1 of the Supporting Information. It is clear to see that all samples show a narrow pore size distribution, suggesting the uniform porosity of the obtained materials, while the sequence of the pore diameter is in the order of MCM-41(DU)-F > MCM-41(DU) > MCM-41(AF). This is probably attributed to the formation of soluble $\mathrm{NH}_{4} \mathrm{~F}$ in the process, which could be easily dissociated, and the released fluorine ions are beneficial for the enlargement of the surfactant micelles.

The physical properties, such as Brunauer-Emmett-Teller (BET) specific surface area, specific pore volume, and average pore diameter, derived from $\mathrm{N}_{2}$ adsorption-desorption measurements are summarized in Table 1. To check the stability of using the photonic waste powder for preparing the mesoporous silicas, duplicate experiments were performed and the BET characterization of the three waste-derived mesoporous samples prepared from two different batches of waste 
powder showed that there was negligible effect on the sample pore structure (see Figure S2 of the Supporting Information). This could be attributed to the fact that the solid waste powder was obtained from the same TFT-LCD plant, which controls their process precisely and stably. One can see that the specific surface area and total pore volume of MCM-41(DU)-F are higher than those of MCM-41(DU), revealing that MCM41(DU)-F possesses superior quality of the mesostructure, and this result is in agreement with the XRD results shown in Figure 2a.

The chemical composition of the obtained MCM-41(DU)-F sample was investigated by X-ray photoelectron spectroscopy (XPS) and energy-dispersive spectrometry (EDS) analyses, and the results are presented in panels $a$ and $b$ of Figure $S 3$ of the Supporting Information. As seen from the spectra, the obtained silica materials contain silicon and oxygen elements and there is no observable impurity, suggesting that highly purified siliceous materials can be obtained by the proposed method in this study. The textural structure was obtained from transmission electron microscopy (TEM) analysis, as shown in panels $\mathrm{c}$ and $\mathrm{d}$ of Figure S3 of the Supporting Information. It can be observed that MCM-41(DU) material shows a poor-organized mesostructure, whereas MCM-41(DU)-F exhibits well-organized hexagonal pore arrangement, implying that the addition of hydrofluoric acid is beneficial for the formation of wellorganized mesoporous silica from waste powder. This is consistent with the results of XRD and BET analyses. In addition, the uniform porosity of obtained MCM-41(DU)-F revealed by TEM analysis is in agreement with the narrow pore size distribution $(\mathrm{BJH})$ determined by $\mathrm{N}_{2}$ adsorptiondesorption measurement. As a result, one can conclude that pure silica materials with ordered mesostructure can be directly recovered from the photonic waste powder at room temperature with the assistance of $\mathrm{CTAB}$, hydrofluoric acid, and ammonium hydroxide.

It could be speculated from the previous results of XRD, BET, and TEM that silica materials with well-organized mesostructure can only be formed when all silicon species were first preactivated by hydrofluoric acid. It was proposed by the reaction eqs $4-8$ that the addition of hydrofluoric acid in the system can first induce the reactive fluorine ions with nonreactive $\mathrm{SiO}_{2}$ in the waste powder and form more $\mathrm{SiF}_{6}{ }^{2-}$ species, which can be rapidly hydrolyzed to more reactive $\mathrm{Si}(\mathrm{OH})_{4}$ and/or $\mathrm{SiO}(\mathrm{OH})_{3}{ }^{-}$anions by adding ammonium hydroxide. Then, well-ordered MCM-41(DU)-F can be obtained via the strong electrostatic force between silicate species and CTAB molecules. On the other hand, if hydrofluoric acid was not added, then the bulk $\mathrm{SiO}_{2}$ particles could not be dissolved and further hydrolyzed into reactive $\mathrm{Si}(\mathrm{OH})_{4}$ and/or $\mathrm{SiO}(\mathrm{OH})_{3}{ }^{-}$anions. Thus, fewer amounts of reactive silicate anions present in the mixture would result in lower specific surface area and pore volume of MCM-41(DU). Besides, the presence of undissolved $\mathrm{SiO}_{2}$ particles as extraframework particles would also disrupt the uniformity of the mesostructure. This is confirmed by the XRD analysis shown in Figure 2b, where MCM-41(DU) shows a stronger intensity of amorphous $\mathrm{SiO}_{2}$ than that of MCM-41(DU)-F.

Application of Recycled Mesoporous Silicas for $\mathrm{CO}_{2}$ Capture. The uniform mesostructure with high surface area, large pore diameter, and pore volume of waste-derived mesoporous silica materials may imply them as ideal supports of TEPA impregnation for $\mathrm{CO}_{2}$ capture. In comparison, the MCM-41 $(\mathrm{NaSi})$ sample synthesized using a pure chemical reagent of sodium silicate is also performed. Thermogravimetric analyses (TG/DTG) are first conducted to evaluate the amount of TEPA loaded on TEPA-impregnated adsorbents. As seen from Figure $4 \mathrm{a}$, all aminated adsorbents show a broad

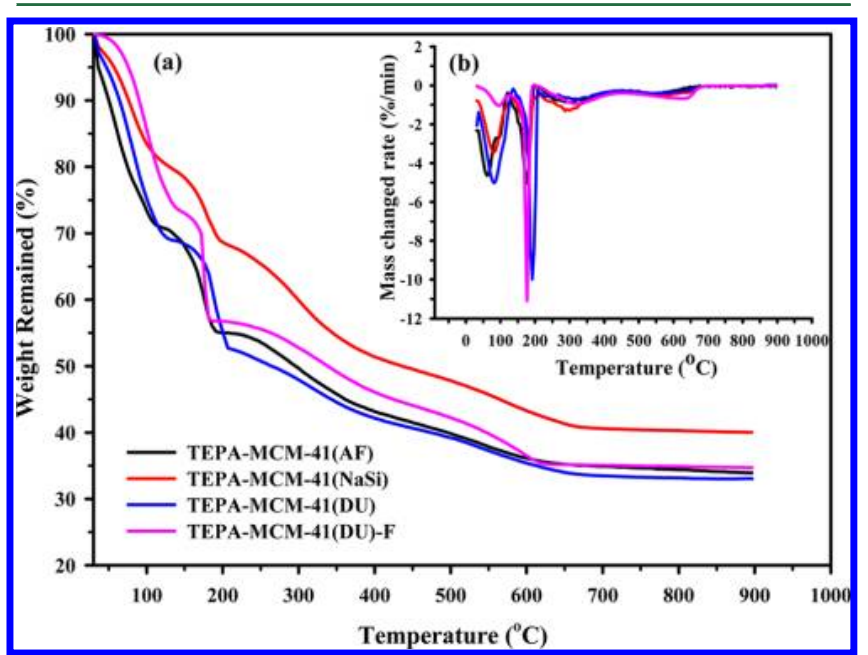

Figure 4. (a) TG and (b) DTG profiles of TEPA-impregnated mesoporous samples.

temperature range of their weight losses. The first weight loss region at $<120{ }^{\circ} \mathrm{C}$ is mainly from the evaporation of the adsorbed water on the surface of the materials. The second weight loss regions at around $120-200{ }^{\circ} \mathrm{C}$ are very significant. It is observed from the DTG profile (Figure $4 \mathrm{~b}$ ) that the maximum mass change rates appeared at around $170-200{ }^{\circ} \mathrm{C}$, and this is mainly attributed to Hofmann elimination of trimethylamine in TEPA-impregnated adsorbents. ${ }^{13}$ The third weight loss regions $\left(200-700{ }^{\circ} \mathrm{C}\right.$ ) are due to the carbon chain $\left(\mathrm{C}-\mathrm{H}_{2}\right)$ decomposition by oxidation processes. ${ }^{13}$

The mass loss observed in the temperature range of 120-700 ${ }^{\circ} \mathrm{C}$ was $40.0 \%$ for TEPA-MCM-41(AF), $42.7 \%$ for TEPAMCM-41(NaSi), $41.1 \%$ for TEPA-MCM-41(DU), and $43.7 \%$ for TEPA-MCM-41(DU)-F, which correspond to 10.57, 11.28, 10.86 , and $11.54 \mathrm{mmol}$ of nitrogen adsorption sites per gram of silica, respectively. It is obvious that similar weight loadings were achieved for all samples.

Figure 5a displays the breakthrough curves of $15 \% \mathrm{CO}_{2}$ adsorption on TEPA-impregnated adsorbents at $60{ }^{\circ} \mathrm{C}$ via the packed column reactor. Initially, all adsorbents could have adsorption efficiencies near $100 \%$. The $\mathrm{CO}_{2}$ adsorption capacities of all adsorbents shown in Table 1 were in a range of $97-120 \mathrm{mg} / \mathrm{g}$ of adsorbent and followed the order of TEPAMCM-41(DU) < TEPA-MCM-41(AF) $\approx$ TEPA-MCM-41$(\mathrm{NaSi})<$ TEPA-MCM-41(DU)-F. It is reported that the adsorption of $\mathrm{CO}_{2}$ by TEPA-impregnated adsorbents was driven mainly between the acidic $\mathrm{CO}_{2}$ molecules and the basic amino groups within the TEPA molecules. Generally, the following chemical reactions are expected to take place when $\mathrm{CO}_{2}$ molecules react with TEPA: ${ }^{1}$

$$
\begin{aligned}
& \mathrm{CO}_{2}+2 \mathrm{RNH}_{2} \rightarrow \mathrm{RNHCOO}^{-}+\mathrm{RNH}_{3}^{+} \\
& \mathrm{CO}_{2}+2 \mathrm{R}_{1} \mathrm{R}_{2} \mathrm{NH} \\
& \rightarrow \mathrm{R}_{1} \mathrm{NHCOO}^{-}+\mathrm{R}_{2} \mathrm{NH}_{2}^{+}\left(\text {or } \mathrm{R}_{2} \mathrm{NCOO}^{-}+\mathrm{R}_{1} \mathrm{NH}_{2}^{+}\right)
\end{aligned}
$$





Figure 5. (a) $\mathrm{CO}_{2}$ breakthrough curves for TEPA-impregnated adsorbents of MCM-41(NaSi), MCM-41(AF), MCM-41(DU), and MCM-41(DU)$\mathrm{F},(\mathrm{b})$ relationship between the amine surface density and $\mathrm{CO}_{2}$ uptake, (c) relationship between the pore diameter of the mesoporous substrates and $\mathrm{CO}_{2}$ uptake, and (d) correlation of the total pore volume of the mesoporous substrates and $\mathrm{CO}_{2}$ uptake at $60{ }^{\circ} \mathrm{C}$.

$$
\begin{aligned}
& \mathrm{CO}_{2}+\mathrm{R}_{2} \mathrm{NH}+\mathrm{RNH}_{2} \\
& \quad \rightarrow \mathrm{R}_{2} \mathrm{NCOO}^{-}+\mathrm{RNH}_{3}^{+}\left(\text {or } \mathrm{RNHCOO}^{-}+\mathrm{R}_{2} \mathrm{NH}_{2}{ }^{+}\right)
\end{aligned}
$$

It is seen that the two amino groups are required to capture one $\mathrm{CO}_{2}$ molecule. As published by Hiyoshi et al., the dense amino groups are more effective as adsorption sites than those isolated on bare silica supports. ${ }^{25}$ Thus, the key parameter affecting the $\mathrm{CO}_{2}$ adsorption by aminated adsorbents is not the surface area but might be the density of amines on the surface of the support.

The surface density of amines in the aminated samples was determined by taking into account the amine loading calculated from TGA analysis and surface area of parent mesoporous support, and the results are plotted in Figure $5 \mathrm{~b}$. It is seen that the sorbent capacity is in proportion with the surface density of amine and follows the order of TEPA-MCM-41(AF) $(112 \mathrm{mg} /$ $\mathrm{g}$ of adsorbent and $6.0 \mathrm{~N}$ atom $\left./ \mathrm{nm}^{2}\right) \approx$ TEPA-MCM-41 $(\mathrm{NaSi})$ $\left(113 \mathrm{mg} / \mathrm{g}\right.$ of adsorbent and $6.2 \mathrm{~N}$ atom $\left./ \mathrm{nm}^{2}\right)<$ TEPA-MCM41 (DU)-F $\left(120 \mathrm{mg} / \mathrm{g}\right.$ of adsorbent and $8.8 \mathrm{~N}$ atom $\left./ \mathrm{nm}^{2}\right)$. However, it is noteworthy that the TEPA-MCM-41(DU) sample showed relatively lower $\mathrm{CO}_{2}$ capacity $(97 \mathrm{mg} / \mathrm{g}$ of adsorbent), even though it exhibits the highest amine surface density $\left(16.5 \mathrm{~N}\right.$ atom $\left./ \mathrm{nm}^{2}\right)$. In this sense, the amino surface density is not the only parameter affecting $\mathrm{CO}_{2}$ adsorption performance in these aminated adsorbents.
In addition to amine surface density, the effect of textural properties of silica support on $\mathrm{CO}_{2}$ adsorption was also studied. Son and co-workers employed a series of 50 wt \% polyethylenimine (PEI)-impregnated mesoporous silicas as adsorbents for $\mathrm{CO}_{2}$ adsorption. ${ }^{26}$ They found that the adsorption capacity was a function of the pore diameter of the bare support and followed the order of MCM-41 (2.8 nm and 1D) < MCM-48 (3.1 nm and 3D) < SBA-15 (5.5 nm and $1 \mathrm{D}) \approx \operatorname{SBA}-16(4.1 \mathrm{~nm}$ and $3 \mathrm{D})<\operatorname{KIT}-6(6.5 \mathrm{~nm}$ and 3D). However, in this study, MCM-41(DU) with a relatively larger pore diameter $(4.4 \mathrm{~nm})$ appears to have lower $\mathrm{CO}_{2}$ uptakes compared to those of MCM-41(AF) (3.0 nm), MCM$41(\mathrm{NaSi})(3.1 \mathrm{~nm})$, and MCM-41(DU)-F (4.5 nm) (Figure 5c). The $\mathrm{CO}_{2}$ capacity, on the other hand, increased with an increasing total pore volume of the silica support. As seen from Figure $5 \mathrm{~d}$, a linear relationship with a correlation coefficient $R^{2}$ $=0.97$ was observed between the total pore volume of the parent silica supports and the $\mathrm{CO}_{2}$ capacity. This clearly indicates that the total pore volume of the bare silica support plays a predominant role in $\mathrm{CO}_{2}$ adsorption rather than the pore diameter.

The density of TEPA is $0.99 \mathrm{~cm}^{3} / \mathrm{g}$, and the total pore volumes of MCM-41(NaSi), MCM-41(AF), MCM-41(DU), and MCM-41(DU)-F are 1.00, 0.99, 0.52 , and $1.10 \mathrm{~cm}^{3} / \mathrm{g}$, respectively. Thus, the maximum theoretical TEPA amounts loaded inside the pore channels were calculated to be 50,50, 34 , and $53 \%$ for MCM-41(NaSi), MCM-41(AF), MCM- 
Table 2. Comparison of the TEPA-Related Adsorbents on Their Synthesis and $\mathrm{CO}_{2}$ Adsorption Performance

\begin{tabular}{|c|c|c|c|}
\hline support & Si precursor & manufacture process & $\begin{array}{c}\text { capacity } \\
\text { (mg of } \mathrm{CO}_{2} / \mathrm{g} \text { of adsorbent) }\end{array}$ \\
\hline $\mathrm{MCM}-41(\mathrm{NaSi})$ & $\begin{array}{l}\text { sodium } \\
\text { silicate }\end{array}$ & hydrothermal method $\left(145^{\circ} \mathrm{C}, 36 \mathrm{~h}\right)$ & $113 \pm 3$ \\
\hline MCM-41(AF) & waste powder & $\begin{array}{l}\text { alkali fusion extraction }\left(550^{\circ} \mathrm{C}, 1 \mathrm{~h}+\text { room temperature, } 24 \mathrm{~h}\right)+\text { hydrothermal method } \\
\left(145^{\circ} \mathrm{C}, 36 \mathrm{~h}\right)\end{array}$ & $112 \pm 3$ \\
\hline MCM-41(DU) & waste powder & direct utilization method (room temperature, $8 \mathrm{~h}$ ) & $97 \pm 6$ \\
\hline $\mathrm{MCM}-41(\mathrm{DU})-\mathrm{F}$ & waste powder & direct utilization method (room temperature, $8 \mathrm{~h}$ ) & $120 \pm 4$ \\
\hline
\end{tabular}

41(DU), and MCM-41(DU)-F, respectively. The pores of MCM-41(DU), MCM-41(AF), and MCM-41(NaSi) were nearly filled with $50 \mathrm{wt} \%$ TEPA; therefore, this may easily result in the blockage of effective adsorption sites and constricted pores, leading to the decrease in adsorption capacity. One can see from the $\mathrm{BJH}$ pore size distribution plots (see Figure S4 of the Supporting Information) that the pores of MCM-41(AF) and MCM-41(NaSi) are diminished significantly after TEPA impregnation. On the contrary, parts of mesopores are still maintained in TEPA-MCM-41(DU)-F, and this could make the gas flow into TEPA-MCM-41(DU)-F more easily, which is beneficial for $\mathrm{CO}_{2}$ adsorption. Thus, more efficient contact between the $\mathrm{CO}_{2}$ gas and the impregnated TEPA could be achieved when a small space is still left insides the pores of the silica support after amine loading.

Lately, $\mathrm{Qi}$ et al. ${ }^{27}$ demonstrated the relationships between the $\mathrm{CO}_{2}$ capacity, amine loadings, and pore structures, including surface area, pore diameter, and total pore volume. They found that the sorbent capacity is more related to the total pore volume of the support than the other parameters. Beyond a critical amine amount, the pore blocking would dramatically occur in the sorbents and, thus, impede adsorption. A similar observation was also found by Yan et al., who found that the sorbent capacity increased with the total pore volume of the parent SBA-15 support. $^{28}$ The results by Qi et al. ${ }^{27}$ and Yan et al. $^{28}$ seem to be consistent with the results of this study. Consequently, one may conclude that, although the dispersion of amino groups on the high surface area materials may increase the adsorption capacity, the pore diameter and pore volume of the support seem to largely influence the adsorption capacity.

Comparison of MCM-41(NaSi), MCM-41(AF), and MCM-41(DU)-F. Table 2 lists the summary on the comparison of TEPA-related materials used for $\mathrm{CO}_{2}$ adsorption in terms of their starting precursors, manufacture process, and sorption capacities. MCM-41(NaSi), which was prepared using pure chemical silicate precursor, can be considered as a promising adsorbent for efficient $\mathrm{CO}_{2}$ capture. However, they may pose problems in practical field applications because of their high cost, and this would be one of the most important considerations for industrial applications.

Generally, a significant portion of the cost to prepare mesoporous silica materials is due to the use of the silicate source because the other required components, i.e., the structure directing agents, such as CTAB surfactants, can be easily recycled using the solvent extraction method under mild conditions $\left(<100{ }^{\circ} \mathrm{C}\right)$, which is obviously more cost-effective and energy-saving than the high-temperature calcination treatment. Commercial fabrication of sodium silicate is associated with high temperature, high pressure, and strong acidity, which is costly, energy-consumed, and non-environmentally friendly. In this respect, avoiding the use of commercial silica precursors for the synthesis of siliceous advanced materials seems to be highly desirable and would be beneficial in terms of cost, environmental impact, and scale-up potential.

The production of valuable silica-related materials from industrial waste exhibits advantages of reducing both the waste production rate and natural resource consumption rate. This has been especially important on using natural resources for the capture of abundant $\mathrm{CO}_{2}$ greenhouse gas. The use of wastederived materials as adsorbents can not only reduce the $\mathrm{CO}_{2}$ greenhouse gas emission but also produce less waste after $\mathrm{CO}_{2}$ adsorption because the adsorbent itself was made from the waste materials. In this regard, the MCM-41(AF), MCM41(DU), and MCM-41(DU)-F adsorbents were produced using photonic industrial silicate waste. Even though MCM$41(\mathrm{AF})$ with an ordered mesostructure and a high $\mathrm{CO}_{2}$ capacity can be obtained, the alkali fusion treatment might not be an economic and energy-effective approach because the process is typically performed under a high temperature of $550{ }^{\circ} \mathrm{C}$.

On the other hand, MCM-41(DU)-F, which has the highest $\mathrm{CO}_{2}$ capacity of $120 \pm 4 \mathrm{mg} / \mathrm{g}$ among all tested adsorbents, can be facilely prepared using photonic industrial waste with the assistance of HF. In photonic and semiconductor industries, $\mathrm{HF}$ is also produced as a liquid waste and, thus, can be reused as a useful resource instead of being wasted. In terms of a greener production of the adsorbent, MCM-41(DU), which did not use $\mathrm{HF}$ in the material synthesis process, may also be a good candidate for $\mathrm{CO}_{2}$ adsorption because it only sacrifices a small amount of the $\mathrm{CO}_{2}$ adsorption capacity.

In conclusion, the proposed manufacture of silica adsorbents from waste silica sources can be processed under roomtemperature conditions, and it is simple, cost-effective, and energy-saving compared to the traditional alkaline fusion method for the extraction of silica from waste resources. This novel process could also be employed for the extraction of silica from other industrial and agricultural wastes and applied as adsorbents or catalysts for a wide range of environmental pollution control applications.

\section{ASSOCIATED CONTENT}

\section{S Supporting Information}

Experimental characterization, $\mathrm{N}_{2}$ physisorption isotherms, $\mathrm{BJH}$ pore size distribution, XPS and EDS spectra, and TEM and SEM images. This material is available free of charge via the Internet at http://pubs.acs.org.

\section{AUTHOR INFORMATION}

\section{Corresponding Author}

*Telephone: +886-3-5731868. Fax: +886-3-5725958. E-mail: hlbai@mail.nctu.edu.tw.

\section{Notes}

The authors declare no competing financial interest. 


\section{ACKNOWLEDGMENTS}

The authors gratefully acknowledge the financial support from the National Science Council, Taiwan, through Grant NSC 982221-E-009-023-MY3.

\section{REFERENCES}

(1) Lin, L.-Y.; Kuo, J.-T.; Bai, H. Silica materials recovered from photonic industrial waste powder: Its extraction, modification, characterization and application. J. Hazard. Mater. 2011, 192, 255262.

(2) Cama, J.; Ayora, C.; Querol, X.; Ganor, J. Dissolution kinetics of synthetic zeolite $\mathrm{NaP1}$ and its implication to zeolite treatment of contaminated waters. Environ. Sci. Technol. 2005, 39, 4871-4877.

(3) Dunens, O. M.; MacKenzie, K. J.; Harris, A. T. Synthesis of multiwalled carbon nanotubes on fly ash derived catalysts. Environ. Sci. Technol. 2009, 43, 7889-7894.

(4) Wang, S. Application of solid ash based catalysts in heterogeneous catalysis. Environ. Sci. Technol. 2008, 42, 7055-7063.

(5) Amrhein, C.; Haghnia, G. H.; Kim, T. S.; Mosher, P. A.; Gagajena, R. C.; Amanios, T.; Torre, L. D. L. Synthesis and properties of zeolites from coal fly ash. Environ. Sci. Technol. 1996, 30, 735-742.

(6) Srinivasan, A.; Grutzeck, M. W. The adsorption of $\mathrm{SO}_{2}$ by zeolites synthesized from fly ash. Environ. Sci. Technol. 1999, 33, 1464-1469.

(7) Lin, C.-F.; Hsi, H.-C. Resource recovery of waste fly ash: Synthesis of zeolite-like materials. Environ. Sci. Technol. 1995, 29, $1109-1117$.

(8) Hui, K. S.; Chao, C. Y. H. Methane emissions abatement by multi-ion-exchanged zeolite A prepared from both commercial-grade zeolite and coal fly ash. Environ. Sci. Technol. 2008, 42, 7392-7397.

(9) Querol, X.; Alastuey, A.; López-Soler, A.; Plana, F.; Andrés, J. M.; Juan, R.; Ferrer, P.; Ruiz, C. R. A fast method for recycling fly ash: Microwave-assisted zeolite synthesis. Environ. Sci. Technol. 1997, 31, $2527-2533$.

(10) Kresge, C. T.; Leonowicz, M. E.; Roth, W. J.; Vartuli, J. C.; Beck, J. S. Ordered mesoporous molecular sieves synthesized by a liquidcrystal template mechanism. Nature 1992, 359, 710-712.

(11) Hung, C.; Bai, H.; Karthik, M. Ordered mesoporous silica particles and Si-MCM-41 for the adsorption of acetone: A comparative study. Sep. Purif. Technol. 2009, 64, 265-272.

(12) Karthik, M.; Lin, L.-Y.; Bai, H. Bifunctional mesoporous Cu-AlMCM-41 materials for the simultaneous catalytic abatement of $\mathrm{NO}_{x}$ and VOCs. Microporous Mesoporous Mater. 2009, 117, 153-160.

(13) Lin, L.-Y.; Bai, H. Continuous generation of mesoporous silica particles via the use of sodium metasilicate precursor and their potential for $\mathrm{CO}_{2}$ capture. Microporous Mesoporous Mater. 2010, 136, $25-32$.

(14) Baccile, N.; Grosso, D.; Sanchez, C. Aerosol generated mesoporous silica particles. J. Mater. Chem. 2003, 13, 3011-3016.

(15) Iskandar, F.; Gradon, L.; Okuyama, K. Control of the morphology of nanostructured particles prepared by the spray drying of a nanoparticle sol. J. Colloid Interface Sci. 2003, 265, 296-303.

(16) Kosuge, K.; Sato, T.; Kikukawa, N.; Takemori, M. Morphological control of rod- and fiberlike SBA-15 type mesoporous silica using water-soluble sodium silicate. Chem. Mater. 2004, 16, 899905.

(17) Jang, H. T.; Park, Y.; Ko, Y. S.; Lee, J. Y.; Margandan, B. Highly siliceous MCM-48 from rice husk ash for $\mathrm{CO}_{2}$ adsorption. Int. J. Greenhouse Gas Control 2009, 3, 545-549.

(18) Misran, H.; Singh, R.; Begum, S.; Yarmo, M. A. Processing of mesoporous silica materials (MCM-41) from coal fly ash. J. Mater. Process. Technol. 2007, 186, 8-13.

(19) Bhagiyalakshmi, M.; Yun, L. J.; Anuradha, R.; Jang, H. T. Synthesis of chloropropylamine grafted mesoporous MCM-41, MCM48 and SBA-15 from rice husk ash: Their application to $\mathrm{CO}_{2}$ chemisorption. J. Porous Mater. 2009, 17, 475-484.
(20) Chandrasekar, G.; You, K.-S.; Ahn, J.-W.; Ahn, W.-S. Synthesis of hexagonal and cubic mesoporous silica using power plant bottom ash. Microporous Mesoporous Mater. 2008, 111, 455-462.

(21) Chang, H.-L.; Chun, C.-M.; Aksay, I. A.; Shih, W.-H. Conversion of fly ash into mesoporous aluminosilicate. Ind. Eng. Chem. Res. 1999, 38, 973-977.

(22) Hui, K. S.; Chao, C. Y. H. Synthesis of MCM-41 from coal fly ash by a green approach: Influence of synthesis pH. J. Hazard. Mater. 2006, 137, 1135-1148.

(23) Mokhonoana, M. P.; Coville, N. J. Synthesis of [Si]-MCM-41 from TEOS and water glass: The water glass-enhanced condensation of TEOS under alkaline conditions. J. Sol-Gel Sci. Technol. 2010, 54, $83-92$.

(24) Sayari, A.; Kruk, M.; Jaroniec, M.; Moudrakovski, I. L. New approaches to pore size engineering of mesoporous silicates. Adv. Mater. 1998, 10, 1376-1379.

(25) Hiyoshi, N.; Yogo, K.; Yashima, T. Adsorption of carbon dioxide on amine modified SBA-15 in the presence of water vapor. Chem. Lett. 2004, 33, 510-511.

(26) Son, W.-J.; Choi, J.-S.; Ahn, W.-S. Adsorptive removal of carbon dioxide using polyethyleneimine-loaded mesoporous silica materials. Microporous Mesoporous Mater. 2008, 113, 31-40.

(27) Qi, G.; Fu, L.; Choi, B.-H.; Giannelis, E.-P. Efficient $\mathrm{CO}_{2}$ sorbents based on silica foam with ultra-large mesopores. Energy Environ. Sci. 2012, 5, 7368-7375.

(28) Yan, X.; Zhang, L.; Zhang, Y.; Yang, G.; Yan, Z. Amine-modified SBA-15: Effect of pore structure on the performance for $\mathrm{CO}_{2}$ capture. Ind. Eng. Chem. Res. 2011, 50, 3220-3226. 